Rev. Est. de Políticas Públicas, 5(1): diciembre 2018 - junio 2019, 33-49

http://dx.doi.org/10.5354/0719-6296.2019.51286

ISSN edición web: 0719-6296

CCopyright 2019: Universidad de Chile, Santiago (Chile)

\title{
Una propuesta para medir la calidad e imagen percibida y su efecto sobre la satisfacción de usuarios en instituciones públicas: Un enfoque PLS-SEM, aplicado a un municipio en Chile
}

\author{
Julio Rojas, Sara Arancibia, Carlos Andrade y Álvaro Ramírez-Alujas \\ Universidad de Chile, Universidad Diego Portales, Universidad Alberto Hurtado y \\ Universidad de Chile
}

\begin{abstract}
Resumen
En la actualidad, los usuarios de instituciones públicas exigen servicios acorde a sus necesidades, esperando una atención de calidad que les permita sentirse satisfechos con el servicio recibido. En Chile, un tema pendiente en la agenda de modernización del Estado es la mejora real de la calidad de servicio en el sector público. Particularmente, a nivel de municipios este proceso ha sido insuficiente y existe escasa evidencia de estudios que midan la percepción de satisfacción usuaria y calidad de servicio. El presente estudio propone un modelo PLS de Ecuaciones Estructurales para medir y explicar los factores que inciden en la calidad percibida y la imagen, y cómo estos factores afectan la satisfacción de los usuarios de servicios de instituciones públicas. El modelo fue aplicado a los servicios entregados por la Municipalidad de la comuna de Lo Prado de la Región Metropolitana de Chile. Los resultados obtenidos muestran que la "Eficiencia en los procedimientos" relativos a la agilidad de los procesos de atención y la "Atención del personal" son los factores que producen el mayor efecto sobre la "Calidad percibida" y la "Satisfacción". El modelo expuesto se proyecta por los autores como una herramienta de gestión que permite apoyar las decisiones de focalización de los recursos disponibles y de mejoras.
\end{abstract}

Palabras clave: Satisfacción, Calidad Percibida, Servicio Público.

A proposal to measure perceived quality and image and their effect on user's satisfaction in public institutions: A PLS-SEM approach, applied to a municipality in Chile

\begin{abstract}
Current users of public institutions demand services that fulfill their needs, expecting quality services they can feel satisfied with. In Chile, one of the greatest pending tasks of the State's modernization objectives is to achieve an actual improvement in the quality of the services offered by the public sector. More specifically, on a municipal level, the process has been insufficient, and there is scarce evidence of research measuring users' perceptions of satisfaction and service quality. This study proposes a PLS model with Structural Ecuations to measure and explain the factors that influence perceived quality and image, and how these factors affect users of public institutions. The model was applied to the services rendered by the Municipality of the borough of Lo Prado in the Metropolitan Region, Chile. The obtained results show that "Efficiency in proceedings" related to the speed in which services are provided and the "Staff Assistance" are the factors that produce the largest effects over "Perceived quality" and "Satisfaction". The authors propose the model described above as a management tool; one that supports decision-making, rationalization of available resources and improvements.
\end{abstract}

Keywords: Satisfaction, Perceived Quality, Public Service.

*Dirección de correspondencia [Correspondence address]:

Julio Rojas, Universidad de Chile

E-mail: rojas.julio45@gmail.com

Fecha recepción: 11 de octubre de 2018

Fecha aceptación: 29 de marzo de 2018 


\section{Introducción}

La Modernización de la Gestión Pública en Chile ha alcanzado una fase de desarrollo lento en los últimos años. Si bien entre 1994 y 2000 se otorgó prioridad a tópicos tales como las relaciones con el usuario y la calidad de servicio en el sector público (Morales, 2014: 421), dichas materias no han sido implementadas con éxito en la mayoría de las instituciones públicas del país (Bernstein e Inostroza, 2010). Ello sigue siendo un tema pendiente en la agenda de modernización del sector público en Chile (Ramírez-Alujas, 2004).

En particular, si nos centramos en los municipios, el proceso de modernización ha sido insuficiente (Núñez, 2006). Pocos municipios en el país han iniciado procesos de certificación ISO 9000 respecto a la calidad de sus servicios municipales y su relación con el usuario, entre otras razones por la inversión requerida y exigencia que ello conlleva, en un espacio donde abundan otras prioridades de gasto.

Lo anterior ocurre pese al compromiso del Estado de Chile por generar un cambio en la calidad de la gestión de gobiernos locales. Por medio del Modelo de Gestión de Calidad de los Servicios Municipales -basado en el modelo de excelencia en la gestión de Baldrige National Quality Program (EE.UU)-, el Estado chileno ha propendido a mejorar diferentes dimensiones de la gestión municipal, entre las cuales se encuentran las referidas a la relación con los usuarios y a la prestación de servicios municipales (Subdere, 2008). No obstante, el inconveniente de este sistema es que se define sólo como un marco referencial para planificar y evaluar desempeño (SUBDERE, 2015), el cual al no ser vinculante, desincentiva su aplicación en municipios cuyos recursos son limitados. Por lo tanto, los gobiernos locales poseen pocos incentivos para la creación de mecanismos que recojan la percepción de los ciudadanos, respecto de la satisfacción usuaria y de la calidad de los servicios municipales.

En términos de investigación relativa a la provisión de servicios municipales, existe escasez de evidencia de estudios realizados por municipios que midan la percepción de satisfacción usuaria y calidad de servicio en Chile. Sin este tipo de análisis resulta difícil el trabajo de identificar los aspectos a mejorar. Por ende, lo que podría constituirse como una oportunidad para mejorar la eficiencia de la gestión se presenta con poco valor (Anderson, 1995).

$\mathrm{Al}$ respecto, ¿qué tipo de experiencias avalan la decisión de medir sistemáticamente la opinión de los usuarios, respecto a los servicios que entrega una organización? Algunas respuestas a esta pregunta provienen desde el sector privado.

Autores como Bloemer y de Ruyter (1999) han sostenido, reflexionando desde el sector privado, que la satisfacción del usuario se convierte en el pilar que sustenta los buenos resultados económicos de una empresa. En el mismo sentido, Jones y Sasser (1995) y Macintosh y Lockshin (1997) han comprobado empíricamente una relación positiva entre satisfacción y la "lealtad" del consumidor, lo cual determinaría una rentabilidad a largo plazo para las compañías. Ante ello, existen cada vez más empresas que buscan satisfacer las demandas de sus clientes para su fidelización, o bien para la atracción de nuevos usuarios. No obstante, ¿qué tipo de beneficio podría adjudicarse una institución pública con este tipo de acciones? En este caso, si bien las ganancias no se presentan como beneficios económicos directos, estas podrían alinearse desde otra perspectiva. Vale decir, reputación y lealtad hacia las autoridades, lo cual podría traducirse en créditos políticos, como el ganar una próxima elección; o, por sobretodo, mejorar la satisfacción de la gente que usa los servicios municipales, en tanto imperativo ético de la gestión pública, por lo cual podría transformarse en una provechosa herramienta de gestión, mejorando los factores que los ciudadanos identifiquen como deficientes y que impactan en su bienestar y calidad de vida en lo cotidiano.

Así como el sector privado tiene el indicador económico como el leitmotiv de su gestión, las instituciones públicas necesitan diseñar herramientas para medir la calidad, eficacia y eficiencia de sus acciones, conforme a cumplir su compromiso con los ciudadanos. De esta manera, podría evaluarse en qué magnitud sus planes y programas son valorados positivamente por la comunidad y logran responder a las expectativas de la ciudadanía.

Dado los pocos incentivos que existen para ejecutar acciones orientadas en este sentido en organizaciones públicas, y desde el reconocimiento y adscripción al imperativo ético que tienen los organismos del Estado por entregar servicios de calidad a los usuarios, es que esta investigación propone la elaboración de un modelo multivariante para medir el nivel de satisfacción de los usuarios de los servicios que ofrece una municipalidad. Esto, mediante una modelización con ecuaciones estructurales que permita determinar los factores que más valora el usuario de una institución pública para percibir satisfacción y 
calidad. Por tanto, el objetivo general consiste en proponer un modelo multivariante que identifique y explique los factores que inciden en la calidad percibida, imagen y satisfacción respecto de los servicios otorgados en la municipalidad por parte de los usuarios, con el fin de mejorar los servicios ofrecidos; administrando y focalizando mejor los recursos de una institución pública; y permitiendo, por otro lado, dirigir acciones que mejoren los servicios proveídos, de modo que el usuario reciba la prestación que merece, trayendo como resultado su satisfacción con la oferta entregada por la institución pública.

Lo anterior conlleva a plantear la siguiente pregunta de investigación: ¿Cuáles son los factores organizacionales que influyen en la percepción de calidad, imagen y satisfacción, respecto de los servicios otorgados por una institución pública?

El modelo propuesto para medir la calidad percibida y satisfacción usuaria se aplicó en torno a la evaluación de cinco $^{1}$ servicios entregados al interior de la Municipalidad de la comuna de Lo Prado², los cuales poseen características similares entre sí, en cuanto a su modo de atención y a los productos que otorgan (atención bajo modalidad front office o atención personalizada mediante un trabajador municipal).

\section{Conceptualización de factores}

A continuación, se presenta la conceptualización de los factores que serán considerados en este estudio y una revisión de la literatura que ha reflexionado sobre modelos en torno a ellos.

\subsection{Satisfacción}

¿Cómo ha definido la bibliografía el concepto de satisfacción de usuarios? Algunos autores como Westbrook y Oliver (1991) han señalado que la satisfacción es el producto de una relación de acciones que se da principalmente en el momento en que el cliente o usuario lleva a cabo una transacción, cuya experiencia de intercambio es comparada con las expectativas que se tenían de aquel proceso. En la misma línea, Churchill y Surprenant (1982) sostienen que la satisfacción será producto de un análi-

\footnotetext{
${ }^{1}$ Los cinco servicios evaluados fueron: Servicio de Licencias de Conducir, Permisos de Circulación, Patentes Comerciales, Área de Protección Social y la Oficina Municipal de Inserción Laboral (OMIL).

${ }^{2}$ Comuna ubicada en el norponiente de la Región Metropolitana de Chile, situada en el lugar $n^{\circ} 100$ en el Índice de Desarrollo Humano, 2003
}

sis del usuario respecto de los factores del proceso de atención, considerándose elementos como el precio, el tiempo de espera, expectativas, agilidad, etc. Westbrook y Oliver (1991) también proponían algo similar, calificando a esta línea de investigación como "cognitiva", cuyo foco se ocupaba de observar la satisfacción como la consecuencia de una evaluación de los atributos del producto/servicio y las emociones generadas por este.

En el sector público, la definición del término se aproxima a las anteriormente señaladas. De acuerdo con Van Ryzin (2006: 601), la satisfacción es entendida como la opinión del ciudadano acerca de un producto o servicio.

Desde esa perspectiva, Cabrera et al. (2010) y de la Peña (2014) coinciden y entienden la satisfacción en el sector público como la calidad percibida respecto de los atributos de un servicio ofrecido, considerando la expectativa que el usuario tenía antes de recibirlo.

Estas definiciones, en su conjunto, se acercan a lo propuesto por Lévy y Varela (2006: 456). Ellos hacen referencia a la satisfacción como "la resultante psicológica de una experiencia de consumo, $[. .$.$] donde$ la medida de satisfacción surge de la consistencia en las respuestas a una serie de cuestiones relativas al grado de bienestar que siente una persona". Dicha definición reúne la mayoría de los aspectos destacados por otros autores por lo que, para efectos de este artículo, se entenderá el concepto de satisfacción como "el bienestar resultante de la experiencia del uso o servicio ofrecido por una institución pública".

\subsection{Calidad del servicio}

Para distinguir el concepto de Calidad de Servicio y satisfacción, Zeithaml y Bitner (2002) formularon un esquema para representar la interacción de la satisfacción de usuarios con la calidad percibida. En primer lugar, proponen que la satisfacción requiere que exista un componente experiencial, mientras que la calidad puede darse sólo a nivel de producto o servicio.

En un sentido amplio, Kotler y Armstrong (2003) definen la calidad como la evaluación de la totalidad de aspectos y características de un producto o servicio que se relacionan con su capacidad para satisfacer las necesidades del cliente.

Los enfoques para medir la calidad con mayor difusión han sido la escala Servqual, ideada por los 
investigadores Parasuraman et al. (1988) y la escala Servpref elaborada por Cronin y Taylor (1992). Dichas escalas entienden a la calidad como un insumo clave para preparar a la organización en sus distintas dimensiones, a fin de alcanzar la satisfacción del usuario (Martínez-Tur et al., 2001).

El aporte de estos enfoques para el estudio de la calidad en el sector público es relevante, principalmente desde lo aportado por Zeithaml et al. (1993), ya que existe una cantidad importante de literatura que emplea el término calidad percibida en base a dicha escala. Desde dicha perspectiva, una serie de trabajos han utilizado las dimensiones de calidad servqual como determinantes de la satisfacción del cliente. Entre ellas se pueden mencionar la empatía ofrecida en la atención por parte de los trabajadores de la organización; la capacidad de respuesta de la institución; Seguridad al momento de la atención; Fiabilidad y sinceridad en el producto o servicio comprometido; y la calidad de los tangibles o instalaciones del lugar.

Ejemplo de ello son los artículos de García (2012), del Ayuntamiento de Madrid (2010) y Guerrero (2014). El primero se refiere al análisis realizado sobre los Servicios Sociales del Ayuntamiento de Murcia. Para ello, la autora destaca tres componentes que conforman la atención en los servicios sociales: "El componente técnico científico de los procesos de producción y aplicación de las prestaciones; los contenidos de la relación interprofesional entre profesionales sociales y usuarios; y las condiciones físicas de los equipamientos" (García, 2012: 13). Por su parte, el Ayuntamiento de Madrid realiza una encuesta de satisfacción respecto a la calidad de los servicios ofrecidos por los Centros de Servicios Sociales de la institución. Las dimensiones medidas en aquella oportunidad fueron aspectos organizativos (cita previa, tiempo de espera, horario); y materiales (accesibilidad al Centro, instalaciones); componentes relacionados al trato personal (comprensión, interés mostrado, claridad de la información y amabilidad); y eficacia percibida (rapidez en la respuesta dada) (Ayuntamiento de Madrid, 2010: 3). Guerrero, por su lado, estudió el perfil social y la percepción sobre calidad de los usuarios de los Centros de Servicios Sociales de Ofra (Santa Cruz de Tenerife).

Adicional a los enfoques anteriores, existe un modelo que ha definido los principales activos para lo que es comprendido como el "buen funcionamiento" de una organización. Alude al modelo de capital intelectual (Bueno y Salmador, 2003; Bueno et al., 2008), el cual según el Equipo de investigación CIC
(2003) se define como el conjunto de activos intangibles que, pese a no estar reflejados en los estados contables, generan o generarán valor para la empresa en el futuro. Vale decir, que en la medida en que éstos sean valorados por el usuario, la calidad percibida por los clientes aumentará (Arancibia et al., 2013).

Por definición, el modelo de capital intelectual para medir intangibles (Alama et al., 2006), se conforma de cuatro componentes: capital humano, capital tecnológico, capital organizativo, y capital relacional, los que se definen de la siguiente forma:

- Capital humano: Es aquel que corresponde a los atributos personales de quienes trabajan en el lugar. Recoge tanto las competencias (conocimientos, habilidades, y actitudes) y valores como la capacidad de aprender y crear de las personas y equipos de trabajo.

- Capital organizativo: Son el conjunto de intangibles que conforman el conocimiento sistematizado, explicitado o internalizado por la empresa que queda en la organización, y que estructuran, a su vez, la actividad organizativa. Comprende la cultura, la estructura organizativa y los procesos.

- Capital tecnológico: Se refiere a los intangibles tecnológicos que tienen relación con el desarrollo de las actividades y funciones del proceso de producción o de prestación de servicios, a los que se dedica la empresa.

- Capital relacional: se refiere al conjunto de vínculos que ésta mantiene con los agentes de su entorno: la relación con usuarios, proveedores, aliados, competidores, organismos, sociedad, etc.

Acorde a todo lo anterior, y para efectos de este trabajo, el concepto de Calidad Percibida se compondrá en función a los criterios que determinan la calidad de servicio propuestos por el modelo Servqual (siempre que estas se acondicionen a las características del servicio a evaluar). Asimismo, se contará con el enfoque de Capital Humano que define los elementos constitutivos de las organizaciones, los que en función de su valoración, podrían elevar la calidad del servicio de cualquier institución.

Teniendo en cuenta que la calidad percibida en el sector público responde a una serie de variables, para efectos de esta investigación este concepto será 
entendido y ajustado según la definición de Lévy y Varela (2006:456), quienes indican que es "la evaluación de la experiencia de consumo, tomando como referencia, normalmente, las expectativas del cliente". En esta investigación, el constructo Calidad Percibida se definirá como "la evaluación del servicio recibido por el municipio, en torno a los distintos atributos que entrega la organización al otorgar un servicio, en función de la experiencia del usuario y de sus expectativas".

\subsection{Imagen}

La imagen es un elemento relevante para entender el bienestar de los ciudadanos en torno a los servicios recibidos (Lauro y Esposito Vinzi, 2004; Gutiérrez et al., 2008). Existen diversos estudios referentes al sector privado que también han incluido dicho factor como determinante de la satisfacción (Arancibia et al., 2011, 2013; Bravo et al., 2009; Ball et al., 2006).

La imagen corresponde al conjunto de rasgos y condiciones objetivas del ser social de una institución. Aquellos elementos reconocidos podrían establecerse como su identidad jurídica y/o funcionamiento legal; estructura organizativa $\mathrm{y} / \mathrm{u}$ operativa; su realidad financiera; y la infraestructura en que se sustenta (Chaves, 2003). Desde este enfoque, las empresas han conseguido que el concepto de imagen se convierta en un activo importante para conseguir permanencia y fidelidad del mercado, preparándolas para enfrentar los constantes cambios que suceden en él. De acuerdo a Lewis y Soureli (2006), la imagen se presenta en el momento en que el usuario concibe una idea de la organización, según la experiencia que este ha tenido. Para medirla, los consumidores han de representar su opinión respecto a los aspectos mencionados por (Chaves, 2003), más algunos otros factores como la preocupación de la organización hacia sus clientes, la confiabilidad y/o la reputación que esta proyecta. Desde allí que el instrumento de medición debe ir en función de los elementos que se mencionan aquí, los cuales son parte de la investigación de autores como Ball et al. (2004) y Bravo et al. (2009). Fueron estos mismos quienes comprobaron la relación causal entre la imagen y la satisfacción, concluyendo que, a mejor evaluación en torno a la imagen, la satisfacción de los usuarios debería aumentar.

Para efectos de este artículo la imagen será definida como "aquello que la institución representa para la sociedad y como aquello que el usuario percibe de la institución pública, en relación a la confianza que proyecta y al prestigio de la organización".

\section{Metodología}

Países de la Unión Europea y otras potencias mundiales como el Reino Unido, Canadá y Estados Unidos, han implementado exitosos modelos de análisis de la satisfacción ciudadana en sus instituciones públicas. Todas estas naciones -por medio de los organismos encargados- han decidido compartir la experiencia, tanto desde el punto de vista metodológico (cómo medir y estudiar la satisfacción) como práctico (ejecución de acciones de mejoras).

Cualquiera sea el método, estos países han buscado establecer las variables que más influyen en la satisfacción de la ciudadanía, a objeto de generar planes que mejoren dichas áreas (AEVAL, 2013). Una de las formas para conseguirlo la explican Van Rysin y Del Pino (2009: 15), quienes exponen la utilidad del método de análisis de impulsores claves (o key drivers analysis). Lo hacen dando a conocer un estudio efectuado en la ciudad de Nueva York, en donde se llevó a cabo una encuesta de satisfacción en la que los ciudadanos debían valorar algunos servicios de la ciudad (protección contra incendios, bibliotecas, parques, policía, autobuses, limpieza de las calles, el metro, escuelas y el estado de las calles). Mediante un análisis de regresión, se descubrió que cuatro de estos servicios - la policía, las escuelas, el estado de las carreteras y el metro- eran los impulsores clave de la satisfacción general de los ciudadanos encuestados (Van Ryzin et al., 2004).

Del mismo modo, existen otros estudios que emplean técnicas novedosas para determinar drivers que impactan en la satisfacción. Por ejemplo, los autores Şandor y Raboca (2007) determinaron mediante regresiones lineales múltiples aquellos servicios municipales que más otorgan satisfacción para los ciudadanos de la ciudad de Cluj-Napoca, Rumania. Asimismo, Alizadeh y Kianfar, 2013) realizaron algo similar, pero utilizando el método de la Teoría de los Conjuntos Aproximados (rough set theory) (Pawlak, 1991). Con este lograron determinar la relevancia de los atributos que entrega el gobierno local de Theran, Irán, para mantener los niveles de satisfacción de su población. Dichos autores midieron la satisfacción ciudadana respecto al desempeño de cinco servicios municipales, evaluando ciertas variables que componían cada uno de ellos.

Pese al aporte de estos estudios, se torna difícil obtener conclusiones con una mirada multidimensional 
a través de estos enfoques, pues ninguno ofrece un esquema de relaciones entre todos los factores que pudiesen explicar un determinado fenómeno.

Sin embargo, existe un método que ofrece identificar las relaciones entre los factores que inciden en la variable dependiente (Satisfacción), el cual se expone a continuación.

\subsection{Método PLS-SEM}

Hair et al. (2013) definen a los modelos de ecuaciones estructurales (Structural Equation Modeling) como métodos multivariantes de segunda generación, cuyo propósito es vincular datos y teoría, donde el conocimiento a priori es incorporado dentro del análisis empírico. El modelo general de ecuaciones estructurales combina el uso de variables observables y variables latentes, mientras que su estructura considera dos modelos denominados "modelo de medida" y "modelo estructural". El modelo de medida define las relaciones entre las variables observables y las variables latentes (estas últimas denominadas constructos), que subyacen a ellas. Por otra parte, el modelo estructural describe o explica única y exclusivamente las relaciones entre las variables latentes o constructos.

Existen dos enfoques para estimar los parámetros de un modelo SEM: El enfoque basado en covarianza (CB-SEM) y el enfoque basado en varianzas (PLSSEM). El primer enfoque es más adecuado cuando el modelo propuesto es apoyado por la teoría y cuando el propósito es estimar los parámetros de las relaciones entre constructos. El segundo enfoque es más apropiado en estudios de alcance exploratorio y útil para predecir las variables latentes dependientes del modelo, maximizando la varianza explicada (R2). Este último tiene la ventaja de permitir explorar algunos constructos y relaciones, además de no exigir muestras con distribución normal (Hair et al., 2013; Henseler et al., 2016).

De acuerdo a lo anterior, es importante enfatizar que el método PLS-SEM resulta pertinente para diseñar y testear un modelo de medición de satisfacción usuaria de los servicios que entregan instituciones públicas. Esto debido a que permite conocer si existe relación significativa hacia el constructo que se desea medir, además de conocer las interrelaciones entre los distintos factores involucrados y los efectos directos e indirectos sobre la calidad percibida, imagen y satisfacción. Finalmente, este método permite conocer el porcentaje de varianza explicado por el modelo.

El método PLS-SEM se ha utilizado en diferentes áreas del conocimiento (Esposito Vinzi y Russolillo, 2010; Dibbern et al., 2004). En esa línea, estos modelos han sido ajustados en artículos que han intentado estudiar la relación entre calidad y satisfacción de usuarios en el ámbito público. Aquellos trabajos han analizado la relación entre los factores que inciden en la satisfacción desde diferentes perspectivas. Por ejemplo, Gutiérrez et al. (2009) en su estudio del Ayuntamiento de Castilla y León, midieron el impacto de tres constructos (aspectos técnicos, aspectos funcionales e imagen) sobre la calidad, y esta sobre la satisfacción. Zhang y Pan (2014), analizan la relación positiva entre la reingeniería de procesos del sector público con la satisfacción de los usuarios; mientras que Kitapci et al. (2014) trabajaron la relación entre las dimensiones de calidad de $\mathrm{Pa}-$ rasuraman et al. (1988) en función de la satisfacción de los usuarios de la industria de salud pública en Turquía.

Asimismo, existen estudios desde el sector privado que también han incorporado el uso de ecuaciones estructurales sobre los mismos factores que inciden en la satisfacción del usuario del sector público. Valdunciel et al. (2007), Miguel-Dávila et al. (2010) y Arancibia et al. (2013) han demostrado que factores relacionados con la atención, la organización y el equipamiento físico, influyen en la calidad del servicio y ésta a su vez sobre la satisfacción.

Particularmente, la investigación de Arancibia et al. (2013) propone tres factores formados por intangibles y un factor de equipamiento físico que influyen sobre la imagen y calidad percibida. En ese estudio se desarrolla un modelo de satisfacción en base a metodologías de ecuaciones estructurales, donde se obtiene que los factores de atención del personal y eficiencia organizativa son los factores que más afectan a la calidad percibida. Por su parte, los factores de atención del personal y eficiencia web son los que más influyen en la imagen. Tanto la calidad percibida como la imagen tienen un impacto considerable sobre la satisfacción del cliente.

Aunque este modelo fue aplicado al sector de servicio de la banca, es un modelo idóneo para ser probado en el ámbito del sector público, dado que abarca los factores que más se ven reflejados en la literatura y se ajusta a la realidad de las instituciones públicas. Así, el modelo de este artículo ha sido probado con datos producidos en la municipalidad de Lo Prado. 


\section{Definición el modelo conceptual}

El modelo que se propone toma en cuenta variables que han sido consideradas en el ámbito público y que se ajustan a modelos organizacionales que brindan servicios. Está basado en su estructura básica en el diseño del modelo de Arancibia et al. (2013). El modelo que se probará no considera el factor de servicios de plataforma tecnológica (aunque a futuro se recomienda considerarlo), pues no en todas las instituciones públicas se ofrece el servicio en línea a los usuarios. Por tanto, se consideró para este trabajo aquellos factores que las instituciones públicas tienen en común para brindar servicios en sus respectivos territorios.

Se definieron dos factores intangibles y un factor tangible, los que se presentan como variables explicativas de la calidad y de la imagen. Además, todas las anteriores mencionadas se presentan como predictoras, conforme a los datos producidos por esta investigación, de la satisfacción. Cabe relevar que, para todos los efectos, este estudio alude únicamente a la idea de "causalidad probable". Ello, dado que los datos producidos obedecen a un único momento en el tiempo y no a estudio de orden longitudinal (Singleton y Straits, 2010).

Los constructos considerados se definen a continuación:

- Atención del personal: Captura el cómo el personal atiende a los usuarios de los servicios que brinda la organización, de acuerdo a la percepción de los mismos. Corresponde a las actitudes, habilidades y conocimientos del personal que atiende a los usuarios, referido a la amabilidad, atención oportuna y clara, que genere confianza respecto a sus labores y que entregue tiempo suficiente en la atención. En síntesis, este factor considera la capacidad e idoneidad del personal, y conocimiento de las tareas que cumple.

- Eficiencia en los Procedimientos: Captura el cómo la organización coordina los procedimientos para brindar un servicio eficiente, de acuerdo a la percepción de los usuarios. Corresponde a agilidad en la entrega del servicio buscado, tiempos de espera razonables en la propia atención, procedimientos en general ágiles y aplicación pertinente de los procedimientos para la realización de un trámite, por parte de los funcionarios. En síntesis, este factor considera el nivel de eficiencia en los procedimientos de la organización.

A su vez, se definirá un factor tangible que considera la infraestructura para la atención en la organización, el cual se denominará:

- Equipamiento y entorno para la atención: Captura si la organización cuenta con las condiciones apropiadas para una atención entendida por los usuarios como adecuada. Corresponde a equipamiento y espacios, como lugares de espera limpios, baños en buen estado, espacios provistos de seguridad y dotados de señalética, etc.

En la Figura 1 se plantea el modelo estructural propuesto, donde las flechas de un constructo a otro representan las relaciones causales. Se plantean tres factores o constructos que inciden directamente en la calidad e imagen percibida, los que inciden directamente en la satisfacción. En total se plantean once hipótesis, las que describen una relación directa y positiva. A saber:

H1. El factor "Atención del personal" influye directa y positivamente en la "Calidad Percibida"

H2. El factor "Atención del personal" influye directa y positivamente en el factor "Satisfacción"

H3. El factor "Atención del personal" influye directa y positivamente en el factor "Imagen Percibida"

H4. El factor "Eficiencia en los procedimientos" influye directa y positivamente en la "Calidad Percibida"

H5. El factor "Eficiencia en los procedimientos" influye directa y positivamente en el factor "Satisfacción"

H6. El factor "Eficiencia en los procedimientos" influye directa y positivamente en el factor "Imagen Percibida"

H7. El factor "Equipamiento y entorno para la atención" influye directa y positivamente en la "Calidad Percibida"

H8. El factor "Equipamiento y entorno para la atención" influye directa y positivamente en el factor "Satisfacción" 
Figura 1: Modelo Conceptual de Satisfacción de Usuarios

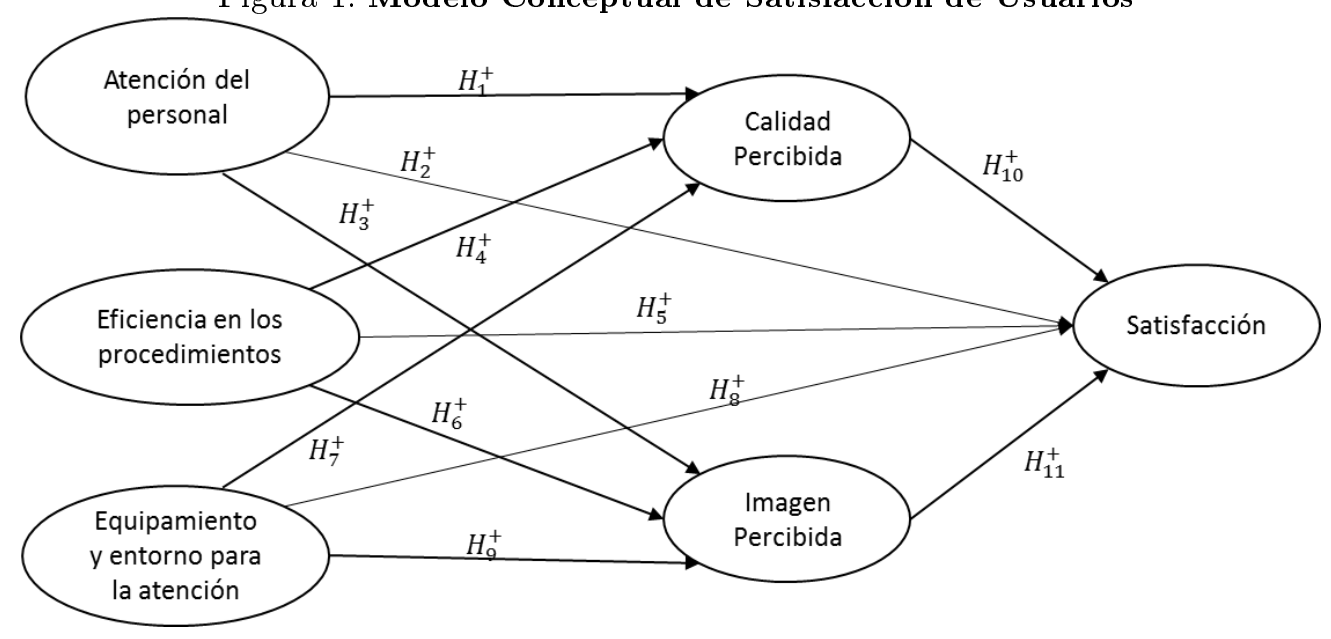

Fuente: Elaboración propia.

H9. El factor "Equipamiento y entorno para la atención" influye directa y positivamente en el factor "Imagen Percibida"

H10. El factor "Calidad Percibida" influye directa y positivamente en la "Satisfacción"

H11. El factor "Imagen Percibida" influye directa y positivamente en el factor "Satisfacción"

Las hipótesis $\mathrm{H} 1, \mathrm{H} 4$ y $\mathrm{H} 7$ son respaldadas por estudios cuyo sustento se basa en los atributos constitutivos de la escala Servqual. Es decir, todo aquel material académico que incluía las dimensiones de calidad de Parasuraman et al. (1988), aplicado para distintos tipos de estudios en el ámbito público (de la Peña, 2014; García, 2012; Guerrero, 2014; Ayuntamiento de Madrid, 2010; Gobierno de Navarra, 2009; Wright et al., 2012. Estos estudios plantean una correlación directa entre los elementos del Capital Humano (CIC, 2002; 2003) y la dimensión "Calidad", por lo cual las trayectorias hipotetizadas en el modelo se respaldan en dicha teoría. A modo de ejemplo, Kal (2014) coinciden en relacionar las dimensiones "empatía, "capacidad de respuesta" y los "tangibles" con los factores de este trabajo "Atención al Personal", "Eficiencia en los procedimientos" y "Equipamiento y entorno para la atención", respectivamente. El mismo caso ocurre con el resto de las hipótesis.

La H2, H5 y H8 indican una relación directa con la satisfacción, cuyo vínculo se ha comprobado en estudios anteriores (Gutiérrez et al., 2009; Gutiérrez et al., 2008; Chatzoglou et al., 2014; Sandor y Raboca, 2007; Van Ryzin et al., 2004; Kitapci et al.,
2014). Şandor y Raboca (2007), por ejemplo, adjudican una relación directa (sin la "Calidad" como constructo intermediario) entre la "personalización" (o customatization) y "confianza" del funcionario, con la satisfacción usuaria. Los dos elementos mencionados entran simultáneamente en la constitución del factor "atención al personal", generando que H2 sea objeto de comprobación. Asimismo, H5 tiene asidero en las investigaciones de Saturno-Hernández et al. (2016) y Kitapci et al. (2014), quienes enfatizan en el rol de componentes relacionados tanto a la empatía y confianza, como a la capacidad de respuesta y la rapidez con la que se atiende a los usuarios. A su vez, este mismo estudio genera evidencia empírica del impacto de los tangiles directamente hacia la satisfacción, dando sustento al testeo de la H8.

Estas investigaciones, por lo tanto, confirman que existe evidencia para afirmar que la satisfacción es explicada en este estudio por la "Calidad", así como también por los factores de "Atención al personal", "Eficiencia en los procedimientos" y "tangibles" por sí solos.

En tanto, las hipótesis H3, H6 y H9 son respaldadas por los estudios de Valdunciel et al. (2007), Miguel-Dávila et al. (2010) y Arancibia et al. (2013). Estos dos últimos aluden al efecto significativo del factor "personal" y "tangibles" sobre la imagen y confianza que una organización puede proyectar, en el contexto de un análisis sobre el sector de la banca. Pese a que ambos estudios destacan la débil relación entre la eficiencia de los procedimientos con la imagen que una institución genera, este trabajo determinará si la gestión en los procesos pudiese influir en 
la imagen del municipio, bajo el supuesto de que una organización eficiente pudiese otorgar reputación e imagen.

La H10, por su parte, es respaldada por la bibliografía referente a los mismos parámetros establecidos por Servqual, más por aquella relacionada al vínculo entre calidad como un constructo obtenido por diferentes variables y satisfacción. Lo anterior, se suma a estudios en el sector público que reflejan la relación directa y positiva entre Calidad y Satisfacción (Chen y Li, 2010; James, 2009; Kelly y Swindell, 2002; Morgeson et al., 2009; Van Ryzin, 004a, 004b, 2006). Lauro y Esposito Vinzi (2004) más Gutiérrez et al. (2008), por ejemplo, establecen teorías y enfoques sobre el comportamiento del cliente en diferentes industrias, aludiendo a que la Calidad percibida (construida por aspectos tales como la atención general entregada por los funcionarios municipales, el desempeño en cuanto a satisfacer requerimientos y la calidad del servicio general) determinan la satisfacción general de los usuarios.

Tanto Lauro y Esposito Vinzi (2004) y Gutiérrez et al. (2008) utilizan constructos y un modelo teórico similar al usado en este estudio, por lo que ambos finalmente sustentan la inclusión de la imagen como predictor de la satisfacción. Según los autores, si el proveedor de los servicios tiene un buen desempeño, esto afecta en su imagen como un servicio eficiente, lo cual finalmente podría afectar a la satisfacción final de la experiencia de servicio. Ergo, un usuario podría calificar su nivel de satisfacción no sólo por la calidad entregada por el servicio, sino que también por la imagen que el usuario concibe respecto a la organización. Dado aquello, la H11 queda como una relación a testear en este modelo.

\section{Instrumento de medición y Mues- treo}

\subsection{Instrumento de medición y trabajo de campo}

Se diseñó una encuesta de modalidad presencial (cara a cara) en función del modelo propuesto y apoyados de la literatura mencionada. La escala de medida de cada pregunta es una escala ordinal de 1 al 7 , siendo 1 totalmente en desacuerdo y 7 totalmente de acuerdo. El instrumento fue aplicado a los usuarios del municipio de la comuna de Lo Prado.

\subsection{Muestra}

El método de muestreo fue no probabilístico, utilizando el muestreo por conveniencia donde los encuestadores tomaron la encuesta de manera voluntaria a los usuarios que asistían al municipio a solicitar un servicio durante un período de cuatro meses (Sampieri et al., 2003).

La muestra consideró un total de 340 encuestas válidas y se aplicó en el edificio Consistorial y el Departamento de Protección Social del municipio. Para el análisis descriptivo de los datos, se utilizó el programa estadístico SPSS 20 y para el desarrollo del modelo estructural el programa SMART PLS 2.0 .

La mayoría de los encuestados son mujeres $(55,6 \%)$, dato que se acerca a la proporción de mujeres de la comuna de Lo Prado. En cuanto a la edad destaca la alta afluencia de público de quienes pertenecen a la categoría de entre 25-44 años (45\%), superando al segmento que se ubica entre los 45-59 años (27,1\%) y a los jóvenes entre 18-24 años (16,4\%). En tanto, las personas de tercera edad $(11,5 \%)$ son quienes menos acuden a los servicios municipales evaluados.

\section{Resultados}

Los resultados se presentan en tres etapas: resultados del ajuste del modelo de medida, resultados del ajuste del modelo estructural, y resultados de los efectos.

\subsection{Resultados del ajuste modelo de medi- da}

El modelo de medida describe cómo cada variable latente es explicada por medio de las variables manifiestas o ítems. Para evaluar la consistencia de los constructos y sus determinados ítems, estos deben cumplir condiciones mínimas de validez y fiabilidad. En este caso, el modelo de medida presenta adecuadas propiedades psicométricas que validan la estimación de las variables latentes, cumpliendo los criterios de validez y fiabilidad que a continuación se describen en la Tabla 1.

La fiabilidad individual del ítem consiste en determinar si las cargas $\lambda$ están asociadas a su respectivo constructo, indicando si el ítem (o variable observada) está correlacionado con las demás cargas. En la Tabla 1 se observan valores de las cargas superio- 
Tabla 1: Indicadores de medida del modelo

\begin{tabular}{|c|c|c|c|c|}
\hline Constructo & Indicador & Fiabilidad individual Carga & AVE & IFC \\
\hline \multirow{5}{*}{ Atención del personal } & P1a & 0,856 & \multirow{5}{*}{0,781} & \multirow{5}{*}{0,930} \\
\hline & P1d & 0,897 & & \\
\hline & P1e & 0,883 & & \\
\hline & P1f & 0,878 & & \\
\hline & P1g & 0,903 & & \\
\hline \multirow{5}{*}{ Eficiencia en los procedimientos } & $\mathrm{P} 2 \mathrm{a}$ & 0,911 & \multirow{5}{*}{0,760} & \multirow{5}{*}{0,920} \\
\hline & $\mathrm{P} 2 \mathrm{~b}$ & 0,866 & & \\
\hline & $\mathrm{P} 2 \mathrm{~d}$ & 0,929 & & \\
\hline & $\mathrm{P} 2 \mathrm{e}$ & 0,800 & & \\
\hline & P2g & 0,845 & & \\
\hline \multirow{4}{*}{$\begin{array}{l}\text { Equipamiento y entorno para la } \\
\text { atención }\end{array}$} & P8a & 0,829 & \multirow{4}{*}{0,663} & \multirow{4}{*}{0,831} \\
\hline & P8b & 0,831 & & \\
\hline & $\mathrm{P} 8 \mathrm{c}$ & 0,793 & & \\
\hline & P8e & 0,804 & & \\
\hline \multirow{4}{*}{ Calidad Percibida } & P9a & 0,920 & \multirow{4}{*}{0,889} & \multirow{4}{*}{0,958} \\
\hline & $\mathrm{P} 9 \mathrm{~b}$ & 0,949 & & \\
\hline & P9c & 0,956 & & \\
\hline & P9d & 0,947 & & \\
\hline \multirow{5}{*}{ Imagen Percibida } & P7a & 0,922 & \multirow{5}{*}{0,817} & \multirow{5}{*}{0,944} \\
\hline & $\mathrm{P} 7 \mathrm{~b}$ & 0,896 & & \\
\hline & P7c & 0,863 & & \\
\hline & $\mathrm{P} 7 \mathrm{~d}$ & 0,938 & & \\
\hline & P7e & 0,898 & & \\
\hline \multirow{4}{*}{ Satisfacción } & P6a & 0,918 & \multirow{4}{*}{0,818} & \multirow{4}{*}{0,926} \\
\hline & $\mathrm{P} 6 \mathrm{~b}$ & 0,917 & & \\
\hline & P6c & 0,931 & & \\
\hline & P6e & 0,850 & & \\
\hline
\end{tabular}

Fuente: Elaboración propia.

res a 0,7 y una carga muy próxima a ese valor, por tanto se verifica la fiabilidad individual del ítem. Un nivel superior a 0,707 o cercano implica que aproximadamente el $50 \%$ de la varianza $\left(\lambda^{2}\right)$ de la variable observada es compartida por el constructo (Cep, 2004).

Además, en todos los constructos el índice de fiabilidad compuesta (IFC) toma valores mayores o iguales a 0,80, cumpliendo lo sugerido por la literatura (Cep, 2004). Este índice verifica si se cumple la consistencia interna de los indicadores de cada constructo. Esto es, si las variables observables están midiendo a la variable latente.

Respecto a la validez convergente, la varianza extraída media (AVE) supera el valor mínimo de 0,5 , lo que significa que el constructo comparte más del $50 \%$ de su varianza con sus indicadores. Su función es evaluar si el conjunto de ítems que miden al constructo están realmente midiendo el concepto y no otro. Por otra parte, según el criterio de Fornell y Larcker (1981) se obtiene que en todos los casos los constructos comparten más varianza con sus indicadores que con el resto de variables latentes, lo que indica que los constructos miden conceptos distintos, es decir, se cumple con la validez discriminante.

\subsection{Resultados de ajuste del modelo estruc- tural}

Para obtener una adecuada interpretación y conclusión del modelo, es necesario realizar la evaluación del modelo estructural, la cual consiste en determinar los coeficientes path $(\beta)$, la varianza explicada $\left(R^{2}\right)$, la relevancia predictiva $\left(Q^{2}\right)$ y el efecto total que tienen los constructos exógenos sobre los constructos endógenos.

En primer lugar, se revisa el valor $t$ de las relaciones entre constructos para verificar si existe una relación estadísticamente significativa. Para lo ante- 
Tabla 2: Valores Beta y resultados Bootstrapping

\section{Relación entre constructos:}

\begin{tabular}{l} 
Calidad Percibida $\rightarrow$ Satisfacción \\
Equipamiento y entorno \\
para la atención $\rightarrow$ Calidad Percibida \\
Equipamiento y entorno \\
para la atención $\rightarrow$ Imagen \\
Equipamiento y entorno \\
para la atención $\rightarrow$ Satisfacción \\
Atención del personal $\rightarrow$ Calidad Percibida \\
Atención del personal $\rightarrow$ Imagen \\
Atención del personal $\rightarrow$ Satisfacción \\
Eficiencia en los \\
procedimientos $\rightarrow$ Calidad Percibida \\
Eficiencia en los \\
procedimientos $\rightarrow$ Imagen \\
Eficiencia en los \\
procedimientos $\rightarrow$ Satisfacción \\
Imagen Percibida $\rightarrow$ Satisfacción \\
\hline Fuente: Elabortion
\end{tabular}

Los coeficientes path o pesos de regresión estandarizados $(\beta)$ miden la fuerza de la relación entre los constructos o de las hipótesis de las relaciones causales planteadas. Chin (1998) propone que para ser considerada una relación significativa la variable predictora debiese explicar al menos el $1,5 \%$ de la variable a predecir. Como se puede ver en la Tabla 3 los cinco constructos explican más del $7 \%$ de la varianza, donde los factores que más aportan a la variabilidad de satisfacción son los factores Eficiencia en los procedimientos, Calidad Percibida y la Imagen.

Por tanto, la variabilidad en el factor de satisfac- ción está siendo explicada en un 82,4\% por el modelo propuesto, el que considera 5 factores tal como se puede ver en la Tabla 3. Para evaluar la relevancia predictiva del modelo, se sigue un procedimiento de Blinfolding mediante el índice $Q^{2}$. En este se omiten parte de los datos para un determinado constructo durante la estimación de los parámetros para a continuación intentar estimar los que se han omitido, usando los parámetros estimados en la primera etapa del proceso (Tenenhaus et al., 2005). Los resultados obtenidos son todos positivos, indicando que se cumple la relevancia predictiva del modelo como se puede ver en la Tabla 4 .

El valor $R^{2}$ hace referencia a la cantidad de la varianza de una variable que es explicada por los constructos dependientes. El umbral de aceptación para estos valores es 0,1 , ya que cifras menores tienen un bajo nivel predictivo (Falk y Miller, 1992). Se puede observar en la Tabla 4 que los tres constructos tienen un alto valor de $R^{2}$, indicando un alto porcentaje de varianza explicado por el modelo. Es decir, la variabilidad en el factor de Calidad Per- 
Tabla 3: Coeficientes path Beta de cada relación con satisfacción

\begin{tabular}{lccc} 
Constructo & $\begin{array}{c}\text { Relación (Beta) } \\
\text { desde el constructo } \\
\text { /hacia Satisfacción }\end{array}$ & $\begin{array}{c}\text { Correlación del } \\
\text { constructo con } \\
\text { Satisfacción (CS) }\end{array}$ & $\begin{array}{c}\text { Porcentaje de } \\
\text { varianza explicada } \\
\text { (S*CS) de } \\
\text { satisfacción }\end{array}$ \\
\hline $\begin{array}{l}\text { Calidad Percibida } \\
\text { Equipamiento y en- }\end{array}$ & 0,2181 & 0,83940 & $18,3 \%$ \\
torno para la atención & 0,1058 & 0,69120 & $7,3 \%$ \\
Atención del personal & 0,1423 & 0,80410 & $11,4 \%$ \\
$\begin{array}{l}\text { Eficiencia en los pro- } \\
\text { cedimientos }\end{array}$ & 0,3325 & 0,84410 & $28,1 \%$ \\
Imagen Percibida & 0,2128 & 0,81240 & $17,3 \%$ \\
Total & & & $\mathbf{8 2 , 4 \%}$ \\
\hline
\end{tabular}

Fuente: Elaboración propia.

Tabla 4: Relevancia predictiva y Varianza explicada por el modelo

\begin{tabular}{lcc}
\multicolumn{1}{c}{ Constructo } & $Q^{2}$ & $R^{2}$ \\
\hline Calidad Percibida & 0,613 & 0,703 \\
Imagen Percibida & 0,509 & 0,635 \\
Satisfacción & 0,663 & 0,824 \\
\hline
\end{tabular}

Fuente: Elaboración propia.

Tabla 5: Efectos totales

\begin{tabular}{lccc}
\multicolumn{1}{c}{ Constructo } & Calidad Percibida & Imagen Percibida & Satisfacción \\
\hline - Calidad Percibida & - & - & 0,2181 \\
- Equipamiento y entorno para & 0,2718 & 0,3153 & 0,2322 \\
la atención & 0,2652 & 0,2905 & 0,2619 \\
- Atención del personal & 0,3992 & 0,2913 & 0,4815 \\
- Eficiencia en los procedi- & - & - & 0,2128 \\
mientos & Fuente: Elaboración propia.
\end{tabular}

Fuente: Elaboración propia.

Rev. Est. de Políticas Públicas, 5(1): diciembre 2018 - junio 2019 
cibida, Imagen Percibida y Satisfacción está siendo explicada respectivamente en un $70,3 \% ; 63,5 \%$ y $82,4 \%$ por el modelo propuesto.

\subsection{Efecto total}

La Tabla 5 indica el efecto total que tiene cada constructo sobre la Calidad Percibida, la Imagen y la Satisfacción de los usuarios de la municipalidad de Lo Prado. Se observa que la Eficiencia en los procedimientos relativos a la agilidad de los procesos de atención y los tiempos reducidos de espera es el que produce el mayor efecto sobre la satisfacción. Le sigue el factor de Atención del personal el que considera la amabilidad, claridad y pertinencia en la información entregada y un tiempo suficiente para la atención por el funcionario. Los otros 3 constructos inciden con efectos similares, entre 0,21 y 0,23 y de manera significativa en la satisfacción. También se observa que la eficiencia en los procedimientos es el que más efecto tiene sobre la calidad percibida, sin embargo, en la Imagen percibida es el Equipamiento y entorno para la atención el de mayor efecto sobre este constructo.

La interpretación del efecto total de cada uno de los factores sobre las variables endógenas, medido en desviaciones estándares, es: si se aumenta en una unidad la percepción de la eficiencia en los procedimientos, entonces la satisfacción aumentará en 0,48 desviaciones estándares. De la misma forma se interpreta cada uno de los efectos.

\section{Conclusiones y futuras líneas de investigación}

Este estudio ha intentado destacar la importancia que tiene la evaluación por parte de la ciudadanía para las organizaciones del sector público. Ello, desde el reconocimiento del imperativo ético que tiene el Estado de entregar servicios de la más alta calidad a sus usuarios. En esta línea, a través de la literatura revisada se ha demostrado que el estudio de la calidad percibida, imagen y satisfacción de los usuarios son aspectos de gran interés de investigación en el contexto de los servicios públicos (AEVAL, 2009, 2013, 2014; Ayuntamiento de Madrid, 2010; Alizadeh y Kianfar, 2013, Brysland y Curry, 2001) y de las organizaciones privadas a nivel internacional (Arancibia et al., 2013; Alama et al., 2006; Bueno y Salmador, 2003; Parasuraman et al., 1990). No obstante, su producción académica en Chile ha sido prácticamente inexistente, en especial en el ám- bito de servicios municipales.

El tipo de modelo propuesto constituye una herramienta de apoyo a la administración pública para identificar prioridades sobre aquellos factores que entregan mayor satisfacción respecto del proceso de atención; apoyar las decisiones de focalización de recursos y de mejoras; además de proponer un modelo de gestión que permita generar valor a las organizaciones en el proceso de atención, cuya principal fuente de información sea la propia opinión de la ciudadanía. Esto último se torna fundamental para cumplir con el mandato de proveer servicios públicos de calidad y al considerar que son los propios ciudadanos los usuarios de los servicios que entrega un organismo público; cuya información podría colaborar en diseñar parte de la planificación estratégica de las instituciones, incorporando indicadores de gestión y desempeño a nivel individual e institucional (Ospina, 2001; del Pino, 2004; del Pino y Díaz, 2011).

Hoy en día, la administración orientada hacia los ciudadanos se transforma en una necesidad para todo tipo de organizaciones. Los habitantes de las democracias desarrolladas han avanzado en pos de disponer de productos y servicios acorde a sus necesidades, en términos, por ejemplo, de una atención de calidad y de un servicio de alto nivel (Van Ryzin y Del Pino, 2009; Parasuraman et al., 1990). Es por esta razón que una herramienta de modelación para medir la percepción de calidad y satisfacción en el sector público cobra cada vez más relevancia, en pos de avanzar hacia la consolidación de un Estado fuerte y robusto que cumpla su rol de primer garante respecto de las necesidades de la ciudadanía. Lo anterior, cobra aún más relevancia considerando la brusca caída en la confianza ciudadana sobre las instituciones públicas en Chile. Según el Séptimo Estudio Nacional de Transparencia (Consejo para la Transparencia, 2015) la evaluación que tienen las personas sobre los organismos públicos es muy baja (3,9 en promedio sobre una escala de 1-10). Tal descontento, puede ser igualmente leído como un posible descontento con la imagen de las instituciones públicas.

Este escenario, no obstante, se convierte en una oportunidad para las instituciones públicas de desarrollar modelos que midan la satisfacción de la ciudadanía. Ello, a modo de fortalecer canales de comunicación que horizontalicen la relación Estadociudadania en pos de la escucha constante de las necesidades de los usarios para, si bien no privativamente, mejorar los servicios públicos proveídos. 
En términos de resultados, en este estudio se observa que el factor de "Eficiencia en los procedimientos" y "Atención del Personal" son los que mayor efecto tienen sobre la Satisfacción y Calidad Percibida, mientras que el factor de tangibles "Equipamiento y entorno para la atención" es el que mayor efecto tiene sobre la Imagen. Estos resultados, en alguna forma se alejan de lo relevado por previas investigaciones (por ejemplo, Bravo et al., 2009). Sin embargo, es plausible pensar que las dinámicas específicas en el sector público difieran de lo relativo al sector privado. Dicho esto, en función de los datos producidos por esta investigación, es esperable para el municipio estudiado que se orienten los recursos hacia estos constructos de forma que el gasto sea eficiente en la medida que cada recurso invertido tenga mayor incidencia en la satisfacción de sus ciudadanos.

En cuanto al modelo, este cuenta con validez y fiabilidad estadística, logrando explicar la varianza del factor Satisfacción en un $82,4 \%$, lo cual confirma su capacidad predictora con base en los datos producidos. Esto significa que lo presentado en este artículo cuenta con la potencialidad para, resguardando las particularidades territoriales, ser replicado en otros modelos de gestión de calidad y satisfacción de distintas reparticiones públicas que brindan servicios. En lo posible, dicha aplicación deberá ser estudiada en base a una muestra probabilística que permita inferir hacia la población total de los usuarios de un determinado servicio.

Si bien el modelo obtiene una alta proporción de varianza, es un desafío para futuras investigaciones incorporar otros determinantes de la satisfacción que no hayan sido recogidos en este estudio. Posiblemente, la integración de un factor que comprenda a los servicios en línea bajo plataformas tecnológicas podría ayudar a incrementar aún más el porcentaje de varianza, pues paulatinamente las reparticiones públicas han comenzado a integrar este tipo de plataformas.

La demanda ciudadana por el uso de estos canales es evidente, por lo cual es un desafío para los municipios en Chile poder incorporar este tipo de tecnologías que propendan a una mejora en los servicios proveídos. En este contexto, ya se han iniciado esfuerzos para promover una red de municipios digitales que contribuyan a este propósito y que, a futuro, debiera ser una variable a incorporar por este tipo de estudios ampliando la comprensión y posibilidades que modelos de este tipo presentan para estudiar la satisfacción de los usuarios con los servi- cios públicos.

\section{Referencias}

(2004). Aplicando en la práctica la técnica pls en la administración de empresas, Murcia.

(2014). Measuring Service Quality in Malaysian Local Government: The SERVQUAL Approach, Malasia.

AEVAL (2009). Guía de evaluación. Modelo EVAM. Modelo de Evaluación, Aprendizaje y Mejora. Ministerio de la Presidencia, Madrid.

AEVAL (2013). El Marco Común de Evaluación. Mejora de las organizaciones públicas por medio de la autoevaluación. Ministerio de la Presidencia, Madrid.

AEVAL (2014). Calidad de los servicios públicos en tiempos de austeridad. Ministerio de la Presidencia, Madrid.

Alama, E., de Castro, M., y López, P. (2006). Capital intelectual. una propuesta para clasificarlo y medirlo. Revista Latinoamericana de Administración, 37:1-16.

Alizadeh, A. y Kianfar, F. (2013). Developing a model for citizens' satisfaction with public sector services based on rough sets theory: a case study of tehran municipality. Technical Gazette, 20(5):95-802.

Anderson, E. (1995). Measuring service quality in a university health clinic. International Journal of Health Care Quality Assurance, 8(2):2-37.

Arancibia, S., Leguina, A., y Espinosa, P. (2013). Factores determinantes en la percepción de la imagen y calidad de servicio y sus efectos en la satisfacción del cliente. un caso aplicado a la banca chilena. Revista de Ciencias Sociales, XIX(2):55-267.

Arancibia, S., Leguina, A., Fernández, a., y Gómez, M. (2011). Focusing resources for customer loyalty: An application to the chilean banking industry. African Journal of Business Management, 6(3):100-1108.

Ayuntamiento de Madrid (2010). Informe resumen sobre la encuesta de satisfaccion de los usuarios de los centros de servicios sociales del ayuntamientio de Madrid. Madrid. 
Ball, D., Coelho, P., y Machás, A. (2004). The role of communication and trust in explaining customer loyalty: an extension to the ecsi model. $E u^{-}$ ropean Journal of Marketing, 38(9):272-1293.

Ball, D., Coelho, P. S., y Vilares, M. J. (2006). Service personalization and loyalty. Journal of services marketing, 20(6):91-403.

Bernstein, F. e Inostroza, J. (2010). Modernización municipal y un sistema de evaluación de su gestión. propuesta de una arquitectura. En Tomicic, V. y García, C., editores, Un mejor Estado para Chile: Propuesta de Modernización y Reforma, pp. 265-314. Santiago.

Bloemer, J. y de Ruyter, K. (1999). Customer loyalty in high and low involvement service settings: The moderating impact of positive emotions. Journal of Marketing Management, 15(4):15-330.

Bravo, R., Montaner, T., y Pina, J. (2009). The role of bank image for customers versus noncustomers. Int. J. Bank. Mark, 27(4):15-334.

Brysland, A. y Curry, A. (2001). Service improvements in public services using servqual. Managing Service Quality, 11(6):89-401.

Bueno, E. y Salmador, M. (2003). Hacia un Modelo Holístico de Capital Intelectual: El Modelo Intellectus. Universidad Autónoma de Madrid, Madrid.

Bueno, E., Salmador, M., y Merino, C. (2008). Génesis, concepto y desarrollo del capital intelectual en la economía del conocimiento: una reflexión sobre el modelo intellectus y sus aplicaciones. Estudios de Economía Aplicada., 266(2):3-64.

Cabrera, F., Donoso, T., Aneas, A., y Del Campo, J. (2010). Valoración de la satisfacción de usuarios de programas sociales: propuesta de un modelo de análisis. Revista de Educación, 351:351-366.

Chatzoglou, P., Chatzoudes, D. V., y Leivaditou, E. (2014). Measuring citizen satisfaction using the servqual approach: the case of the 'hellenic post'. Procedia Economics and Finance, 9:349 -360 .

Chaves, N. (2003). La imagen corporativa. Gili, Barcelona.

Chen, Z. y Li, D. a. (2010). Citizen attitudes toward local government public services: A comparative analysis between the city of xiamen and the city of phoenix. Public Performance and Management Review, 34:221-235.

Churchill, G. y Surprenant, C. (1982). An investigation into determinants on consumer satisfaction. Journal of Marketing Research, 29:491504.

CIC (2002). Guias y Directrices de Utilización del Modelo Intellectus. Centro de Investigación sobre la Sociedad del Conocimiento, Madrid.

CIC (2003). Quinto número de la serie de Documentos Intellectus: Medición y Gestión del Capital Intelectual. Centro de Investigación sobre la Sociedad del Conocimiento, Madrid.

Consejo para la Transparencia (2015). Estudio Nacional de Transparencia. Séptima edición. Market Opinion Research International, Santiago.

Cronin, J. y Taylor, S. (1992). Measuring service quality - a reexamination and extension. Journal of Marketing, 26(july):5-68.

Şandor, S. y Raboca, H. (2007). Determinants and outcomes of citizens' satisfaction with public services in cluj - napoca. Transylvanian Review of Administrative Sciences, October(21):03112.

de la Peña, E. (2014). Análisis de la satisfacción y de la calidad percibida por las personas atendidas en los servicios sociales comunitarios del centro municipal de servicios sociales delicias del ayuntamiento de zaragoza. Cuadernos de Trabajo Social, 27(1):15-125.

del Pino, E. (2004). ¿por qué importan los ciudadanos? tipos de actitudes y consecuencias para el modelo de administración y la vitalidad democrática. Reforma y Democracia. Revista del CLAD, 29:55- 94 .

del Pino, E. y Díaz, J. (2011). Lecciones aprendidas desde la experiencia española de análisis de la percepción ciudadana de los servicios públicos. Reforma y Democracia. Revista del CLAD, 49:57-184.

Dibbern, J., T, G., R., H., y Jayatilaka, B. (2004). Information systems outsourcing: a survey and analysis of the literature. ACM SIGMIS Database, 35:4-102.

Esposito Vinzi, V. y Russolillo, G. (2010). Partial least squares path modeling and regression. En E. Wegman, Y. S. y Scott, D., editores, Wiley 
interdisciplinary reviews: computational. Wiley, New York.

Falk, F. y Miller, N. (1992). A Primer for Soft Modeling. University of Akron Press, Akron.

Fornell, C. y Larcker, D. (1981). Structural equation models with unobservable variables and measurement error: Algebra and statistics. Journal of Marketing Research, 18(3):82-388.

García, G. (2012). Aproximación teórica sobre la calidad percibida en los servicios sociales. Revista de Trabajo Social de Murcia, 17:11-15.

Gobierno de Navarra (2009). Guía para medir Satisfaacción respecto a los servicios prestados. Gobierno de Navarra, Pamplona.

Guerrero, M. (2014). Estudio sobre el perfil social y la percepción de la calidad de las personas usuarias de los Centros de Servicios Social de Ofra. Universidad de La Laguna, Tenerife.

Gutiérrez, P., Cuesta, P., y Vázquez, J. (2008). Determinantes de la satisfacción de los ciudadanos en las administraciones públicas locales. Revista cuatrimestral de las Facultades de Derecho y Ciencias Económicas y Empresariales, 75:91313.

Gutiérrez, P., Vásquez, J., Vaughan, R., y Edwards, J. (2009). The transformation of municipal services: towards quality in the public sector. Theoretical \& Applied Economics, 16:3-16.

Hair, J., Ringle, C. M., y Sarstedt, M. (2013). Editorial-partial least squares structural equation modeling: rigorous applications, better results and higher acceptance. Long Range Planning, 46(1):2-12.

Henseler, J., Hubona, G., y Ash Ray, P. (2016). Using pls path modeling in new technology research: updated guidelines. Industrial Management 6 Data Systems, 116(1):-20.

James, O. (2009). Evaluating the expectations disconfirmation and expectations anchoring approaches to citizen satisfaction with local public. Journal of Public Administration Research and Theory, 19:107-123.

Jones, T. y Sasser, E. (1995). El por qué desertan los clientes satisfechos. Harvard Business Review, Noviembre-Diciembre:2-25.
Kelly, J. M. y Swindell, D. (2002). Service quality variation across urban space: First steps toward a model of citizen satisfaction. Journal of Urban Affairs, 24(3):71-288.

Kitapci, O., Akdogan, C., y Dortyol, I. (2014). The impact of service quality dimensions on patient satisfaction, repurchase intentions and word-ofmouth communication in the public healthcare industry. Social and Behavioral Sciences, 148:161 - 169 .

Kotler, P. y Armstrong, G. (2003). Fundamentos de marketing. McgrawHill, México D.F.

Lauro, C. y Esposito Vinzi, V. (2004). Some contributions to PLS Path Modeling and a system for the European Customer Satisfaction. Dipartimento di Matematica e Statistica Universita Federico II di Napoli, Napoli.

Lévy, J.-P. y Varela, J. (2006). Modelización con Estructuras de Covarianzas en Ciencias. Ediciones Netbiblo, Barcelona.

Lewis, B. y Soureli, M. (2006). The antecedents of consumer loyalty in retail banking. Journal of Consumer Behaviour, 15:15-31.

Macintosh, G. y Lockshin, L. (1997). Retail relationships and store loyalty: A multilevel perspective. International Journal of Research in Marketing, 14(5):87-497.

Martínez-Tur, V., Peiró, J., y José, R. (2001). Calidad de servicio y satisfacción. Síntesis, Madrid.

Miguel-Dávila, J., Cabeza-García, L., Valdunciel, L., y Florez, M. (2010). Operations in banking: the service quality and effects on satisfaction and loyalty. Service. Ind. J., 30(13):163-2182.

Morales, M. (2014). Nueva gestión pública en chile: Orígenes y efectos. Revista de Ciencia Política, $34(2): 17-438$.

Morgeson, I., Forrest, V., y Mithas, S. (2009). Does e-government measure up to e-business?. comparing end user perceptions of us federal government and e-business web sites. Public Administration Review, 69(4):40-752.

Núñez, R. (2006). Realidad y desafíos del municipio chileno. Universidad Autónoma de Chile, Santiago de Chile.

Ospina, S. (2001). Evaluación de la gestión pública: conceptos y aplicaciones en el caso latinoamericano. Reforma y Democracia. Revista del CLAD, 19:1-19. 
Parasuraman, A., Zeithaml, V., y Berry, L. (1988). Servqual: A multiple-item scale for measuring consumer perceptions of service quality.

Parasuraman, A., Zeithaml, V., y Berry, L. (1990). Delivering Quality Service - Balancing Customer Perceptions and Expectations. The Free Press, New York.

Pawlak, Z. (1991). Rough sets: theoretical aspects of reasoning about data. Kluwer Academic Publishing, Dordrecht.

Ramírez-Alujas, a. (2004). El proceso de reforma del Estado y modernización de la gestión pública en Chile. Lecciones, experiencias y aprendizajes (1990-2003. Instituto Nacional de Administración Pública (INAP), Ministerio de Administraciones Públicas (IMAP, Madrid.

Sampieri, R., Collado, C., y Lucio, P. (2003). Metodología de la Investigación. McGraw-Hill Interamericana, México, D. F.

Saturno-Hernández, P. J., Gutiérrez-Reyes, J. P., Vieyra-Romero, W. I., Romero-Martínez, M., O'Shea-Cuevas, G. J., Lozano-Herrera, J., Tavera-Martínez, S., y Hernández-Ávila, M. (2016). Satisfacción y calidad percibida de afiliados al sistema de protección social en salud de méxico. fundamentos metodológicos. Salud pública de méxico, 58(6):85-693.

Singleton, R. A. y Straits, B. C. (2010). Approaches to social research. Oxford University Press, New York,.

Subdere (2008). Modelo de gestión de calidad de los servicios municipales. Ministerio del Interior, Santiago.

SUBDERE (2015). Modelos de Gestión de Calidad Municipal. Santiago de Chile.

Tenenhaus, M., Esposito, V., Chatelin, Y.-M., y Lauro, C. (2005). Pls path modeling. Computational Statistics 83 Data Analysis, 48:159 205.

Valdunciel, L., Florez, M., y Miguel, J. (2007). Análisis de la calidad del servicio que prestan las entidades bancarias y su repercusión en la satisfacción del cliente y la lealtad hacia la entidad. Revista Asturiana de Economía, 38(1):9-99.

Van Ryzin, G. (2004a). Expectations, performance, and citizen satisfaction with urban services. Journal of Policy Analysis and Management, 23:433-448.
Van Ryzin, G. (2004b). The measurement of overall citizen satisfaction. Public Performance and Management Review, 27:9-28.

Van Ryzin, G. (2006). Testing the expectancy desconfirmation model of citizen satisfaction with local government. Journal of Public Administration Research and Theory, 16:599-611.

Van Ryzin, G. y Del Pino, E. (2009). Cómo escuchar, cómo aprender, cómo responder: las encuestas ciudadanas como una herramienta para la reinvención del gobierno. Ministerio de la Presidencia, Madrid.

Van Ryzin, G., Muzzio, D., e Immerwahr, S. (2004). Measuring street cleanliness: A comparison of new york city's scorecard and results of a citizen survey. Public Administration Review, 68(2):95-303.

Westbrook, R. y Oliver, R. (1991). The dimensionality of consumption emotion patterns and consumer satisfaction. U Journal of Consumer Research $U, 18(1): 4-91$.

Wright, G., Chew, C., y Hine, A. (2012). The relevance and efficacy of marketing in public and non-profit service management. Public Management Review, 14(4):33-450.

Zeithaml, V., Berry, L., y Parasuraman, A. (1993). The nature and determinants of customer expectations of service. Journal of the Academy of Marketing Science, 21:1-12.

Zeithaml, V. y Bitner, M. (2002). Marketing de Servicios: Un enfoque de integración del cliente a la empresa. McGraw-Hill, Interamericana Editores, México.

Zhang, L. y Pan, L. (2014). Measuring public satisfaction for government process reengineering. PACIS, Chengdu. 